\title{
Evaluating the Effects of Different Ischaemia Techniques on Functional Outcomes of Laparoscopic Partial Nephrectomy
}

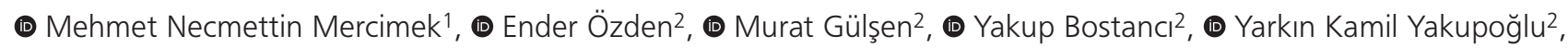 \\ (1) Şaban Sarıkaya² \\ 1 Samsun Liv Hospital, Clinic of Urology, Samsun, Turkey \\ 2 Ondokuz Mayıs University Faculty of Medicine, Department of Urology, Samsun, Turkey
}

\begin{abstract}
Objective: This study aimed to investigate the influence of different ischaemia techniques on short-term and one year renal function following laparoscopic partial nephrectomy (LPN).

Materials and Methods: Data of 359 patients who underwent LPN between November 2009 and April 2018 were reviewed, retrospectively. A total of 287 patients were included in the study. Patients were divided into 4 groups according to type of ischaemia as follows: group A ( $n=33$ ) was warm ischaemia time (WIT) >20 minute, group B ( $n=202)$ was WIT $\leq 20$ minute, group C $(n=16)$ was selective arterial clamping and group $D(n=36)$ was off-clamp. Demographic data, preoperative and postoperative outcomes were compared. The estimated glomerular filtration rate (eGFR) was calculated using the Chronic Kidney Disease Epidemiology Collaboration equation. Change in eGFR according to group and time was evaluated using two-way analysis of variance. P values $<0.05$ were adopted as significant. Results: The mean tumour size was 46, 34, 36 and $25 \mathrm{~mm}(p=0.001)$, and operation time was 123, 92, 100 and 79 minute ( $p=0.001)$ for groups $A, B, C$ and $D$, respectively. There was no need for open surgery in any of the patients. Blood loss, intraoperative and postoperative complication rates were found to be nonsignificantly different ( $p>0.05$ ) between groups. Only 4 patients in group B had positive surgical margin. The percentage of relative eGFR (\% of $\Delta$ eGFR) decline 1 year after LPN was significantly ( $p=0.001$ ) higher in the ischaemic groups ( $A$ and $B: 9.3 \%$ and $7.5 \%)$ compared to the zero-ischaemia groups (C and $D: 5 \%$ and $3.7 \%$ ). Conclusion: This study revealed that after LPN, cumulative renal function decreases to a certain extent regardless of the technique. However, it was found that cumulative renal functions are better preserved when ischaemia time is reduced to $\leq 20$ min or eliminated.
\end{abstract}

Keywords: Ischaemia, laparoscopy, partial nephrectomy, selective ischaemia, zero-ischaemia

\section{Introduction}

Partial nephrectomy (PN) is the gold standard treatment modality for patients with kidney confined renal tumours (if technically feasible) since it ensures similar oncological outcomes and better renal function (RF) preservation compared to radical nephrectomy. Nephron sparing surgery (NSS) preserves RF, decreases the risk of cardiovascular mortality due to chronic renal failure and provides a crucial survival advantage (1).

Postoperative RF status is associated with quality (age, gender, baseline RF and comorbidities), quantity (the amount of healthy kidney tissue preserved) of the renal parenchyma, as well as ischaemia time of one of the essential modifiable risk factors affecting preserved renal parenchyma and postoperative RF (2).
The hypothesis of 'every minute counts' under ischaemia by Thompson et al. (3) led to the advancement in various PN techniques that helped to minimise or eliminate the warm ischaemia time (WIT), although their cut-off limit is still unclear. These techniques include early unclamping, selective arterial clamping and off-clamping. The contribution of laparoscopic and robot-assisted surgeries to this rapid development in the era of minimally invasive NSS cannot be underestimated. Although each technique has its own advantages and disadvantages, open, laparoscopic and robot-assisted approaches produce similar functional and oncologic outcomes (4).

In this article, we aimed to evaluate the effect of different laparoscopic partial nephrectomy (LPN) techniques including global ischaemia, selective-clamp and off-clamp on functional and oncological outcomes.

Cite this article as: Mercimek MN, Özden E, Gülşen M, Bostancı Y, Yakupoğlu YK, Sarıkaya Ş. Evaluating the Effects of Different Ischaemia Techniques on Functional Outcomes of Laparoscopic Partial Nephrectomy. Bull Urooncol 2020;19(3):124-129

Address for Correspondence: Mehmet Necmettin Mercimek, Samsun Liv Hospital, Clinic of Urology, Samsun, Turkey Phone: +90 3629998000 E-mail: m.n.mercimek@hotmail.com ORCID-ID: orcid.org/0000-0002-0680-4451 


\section{Materials and Methods}

The study has been approved by the Ondokuz Mayıs University Clinical Research Ethics Committee (OMU KAEK 14.02.2019/151), and conducted in conformity with the Declaration of Helsinki in 1995. Written informed consent were obtained from all patients included in the study.

Medical records of 351 (out of 359) patients with the complete data who underwent LPN from November 2009 to April 2018 and followed-up at a tertiary care university hospital, department of urology were analysed, retrospectively. Prospectively stored patients' data were retrieved from the hospital's database. All LPNs were performed by the same surgeon.

Patients with solitary kidney $(n=10)$, horseshoe kidney $(n=4)$, pelvic ectopic kidney $(n=2)$, graft kidney $(n=2)$, multiple renal tumours in a single kidney $(n=3)$, bilateral renal tumours $(n=8$, 16 procedures) and patients $<18$ years of age $(n=3)$ were excluded in this study. In addition, patients who without a one year follow-up $(n=32)$ were also excluded from this study. A total of 287 patients with contralateral functioning kidney were included in this study. Patients were grouped into 4 according to the type of ischaemia as follows: group $A(n=33)$ was WIT $>20$ minute, group $B(n=202)$ was WIT $\leq 20$ minute, group $C(n=16)$ was selective-clamp and group $D(n=36)$ was off-clamp.

Demographic characteristics of patients such as age, gender, body mass index (BMI); and systemic diseases such as Diabetes Mellitus, hypertension and coronary artery disease were recorded. Medical data of patients including tumours size, lateralisation of the tumour, surgical technique, operation time (OT), estimated blood loss (EBL), WIT, preoperative and postoperative haemoglobin values, as well as preoperative and postoperative $\left(1^{\text {st }}\right.$ day, $1^{\text {st }}$ month, $6^{\text {th }}$ month and $1^{\text {st }}$ year) serum creatinine levels at were also recorded. Estimated glomerular filtration rate (eGFR) was calculated using the Chronic Kidney Disease Epidemiology Collaboration (CKD-EPI) equation (5). Clinical diagnosis was done by radiological imaging techniques. Magnetic resonance imaging and/or triphasic contrast-enhanced computed tomography was used to analyse the tumour anatomy. Tumour complexity was evaluated by radius of the tumour size, exophytic, nearness to collecting system, anterior, location (RENAL) nephrometry score (6). The final decision on how to perform LPN was based on renal vascular anatomy, RENAL Nephrometry score (RNS), tumour characteristics, vascular anatomy of the kidney, as well as the surgeon's preference or discretion. Intraoperative and postoperative complications, length of hospital stay, final pathology outcomes and surgical margin status were recorded. Furthermore, postoperative complications were graded according to the modified ClavienDindo classification system (I-V) (7).

\section{Surgical Techniques}

Although the patient position and trocar configuration differed according to the transperitoneal (8) and retroperitoneal approach (9), both techniques were applied as previously described in the literature. The renal artery, renal vein and ureter were dissected and then isolated with vascular silicon tapes. The kidney was released from the surrounding tissues as much as possible, and care was taken to ensure the preservation of perirenal fat tissue on the tumour. Laparoscopic ultrasound was used to detect the tumour mass and thus the surgical margin. Monopolar hook was used to score the surgical margin. The renal tumours were completely excised using cold scissors, with a thin negative margin. The tumour bed was sutured in two layers supported with hem-o-lock clips. A great effort was made to secure the remnant renal parenchyma, and prevent bleeding during tumour bed control. The specimen was extracted in an endo-bag, and a drain was placed in the surgical field.

Global ischaemia technique: The main renal artery and vein were dissected and isolated, and then controlled using laparoscopic bulldog clamps, separately.

Selective-clamp technique: The main renal and segmental renal arteries and also the renal vein was dissected and isolated. Afterwards, the segmental artery was controlled with a bulldog clamp. The indocyanine green (ICG) with near-infrared fluorescence imaging was implemented to specify resection margins of the tumour after control of the segmental artery (10).

Off-clamp technique: The main renal artery and vein were dissected and isolated, however, neither hilar nor segmental vessels were clamped in any of the cases. All LPN procedures were performed with WIT of zero (11).

\section{Statistical Analysis}

Data was analysed using Statistics Package for Social Sciences version 24 (IBM SPSS $®$, Armonk, NY). Test for normal distribution was done by the Shapiro-Wilk test. One-Way analysis of variance and independent samples t-test were used for the comparison of normally distributed data. Post-hoc multiple comparisons was done using Tukey HSD test. Generalised linear models were used to determine whether temporal changes of eGFR and $\triangle \mathrm{eGFR}$ values were influence by the groups. Categorical data were analysed using chi-square test. Analysis results were presented as mean \pm standard deviation for quantitative data, and presented as frequency (percentage) for categorical data. $P$ value $<0.05$ was adopted as significant.

\section{Results}

A total of 287 (79.9\%) patients who underwent LPN were included in the study. The demographic data and clinical tumour characteristics of the patients in each group (A-D) are summarised in Table 1 . The age distribution, gender, BMI, American Society of Anesthesiologists score, tumour laterality and tumour localisation were non-significantly $(p>0.05)$ different between the groups. However, the mean tumour size and RNS were statistically different between the groups.

Intraoperative variables, perioperative and postoperative complications are shown in Table 2. While the mean OT in group A was statistically different when compared to groups B and D, it was non-significantly different when compared to group $C$ $(p=0.074)$. In terms of WIT, a statistically significant difference was found between group $A$ and $B$, and between group $B$ and $C(p=0.001)$, while WIT was non-significantly different between group $B$ and $C(p=0.923)$. Since WIT in group D was zero, no statistical comparison was made with respect to other groups. Although the mean EBL was highest in group D, it did not differ when compared to the other groups. 


\begin{tabular}{|c|c|c|c|c|c|}
\hline Variable* & $A(n=33)$ & $B(n=202)$ & $C(n=16)$ & $D(n=36)$ & 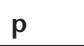 \\
\hline Age, years & $52.82 \pm 10.6$ & $54.77 \pm 12.1$ & $56.56 \pm 11.9$ & $57.61 \pm 13.9$ & 0.391 \\
\hline \multicolumn{6}{|l|}{ Gender } \\
\hline Male & $20(60.6)$ & $119(58.9)$ & $9(56.3)$ & $19(52.8)$ & \multirow{2}{*}{0.901} \\
\hline Female & $13(39.4)$ & $83(41.1)$ & $7(43.8)$ & $17(47.2)$ & \\
\hline $\mathrm{BMI}\left(\mathrm{kg} / \mathrm{m}^{2}\right)$ & $27.18 \pm 3.1$ & $27.47 \pm 3.5$ & $26.20 \pm 2.7$ & $26.75 \pm 3.0$ & 0.378 \\
\hline \multicolumn{6}{|l|}{ DM } \\
\hline Yes & $9(27.3)$ & $34(16.8)$ & $8(50)$ & $6(16.7)$ & \multirow{2}{*}{0.009} \\
\hline No & $24(72.7)$ & $168(83.2)$ & $8(50)$ & $30(83.3)$ & \\
\hline \multicolumn{6}{|l|}{ HT } \\
\hline Yes & $9(27.3)$ & $91(45)$ & $11(68.8)$ & $15(41.7)$ & \multirow{2}{*}{0.048} \\
\hline No & $24(72.7)$ & $111(55)$ & $5(31.3)$ & $21(58.3)$ & \\
\hline \multicolumn{6}{|l|}{ CAD } \\
\hline Yes & $3(9.1)$ & $31(15.3)$ & $3(18.8)$ & $6(16.7)$ & \multirow{2}{*}{0.758} \\
\hline No & 30 (90.9) & $171(84.7)$ & $13(81.3)$ & $30(83.3)$ & \\
\hline \multicolumn{6}{|l|}{ ASA score } \\
\hline 1 & $12(36.4)$ & $72(35.6)$ & $3(18.8)$ & $11(30.6)$ & \multirow{3}{*}{0.501} \\
\hline 2 & $19(57.6)$ & $102(50.5)$ & $10(62.5)$ & $22(61.1)$ & \\
\hline 3 & $2(6.1)$ & $28(13.9)$ & $3(18.8)$ & $3(8.3)$ & \\
\hline \multicolumn{6}{|l|}{ Side } \\
\hline Right & $19(57.6)$ & $97(48)$ & $8(50)$ & $16(44.4)$ & \multirow{2}{*}{0.718} \\
\hline Left & $14(42.4)$ & $105(52)$ & $8(50)$ & $20(55.6)$ & \\
\hline \multicolumn{6}{|l|}{ Localisation } \\
\hline Central & $13(39.4)$ & $80(39.6)$ & $7(43.8)$ & $12(33.3)$ & \multirow{2}{*}{0.880} \\
\hline Polar & $20(60.6)$ & $122(60.4)$ & $9(56.3)$ & $24(66.7)$ & \\
\hline Tumour size, mm & $46.12 \pm 24.6^{a}$ & $34.21 \pm 13.4^{\mathrm{b}}$ & $36.19 \pm 16.7^{\mathrm{abc}}$ & $25.67 \pm 12.79^{c}$ & 0.001 \\
\hline RNS & $7.85 \pm 1.9^{\mathrm{a}}$ & $6.64 \pm 1.6 b$ & $6.44 \pm 1.8^{\mathrm{bc}}$ & $5.50 \pm 1.1^{c}$ & 0.001 \\
\hline \multicolumn{6}{|l|}{ RNS group } \\
\hline Low (4-6) & $8(24.2)$ & $105(52)$ & $9(56.3)$ & $29(80.6)$ & \multirow{3}{*}{0.001} \\
\hline Intermediate (7-9) & $19(57.6)$ & $84(41.6)$ & $6(37.5)$ & $7(19.4)$ & \\
\hline High (10-12) & $6(18.2)$ & $13(6.4)$ & $1(6.3)$ & - & \\
\hline \multicolumn{6}{|c|}{$\begin{array}{l}\text { A: WIT > } 20 \text { min, B: WIT } \leq 20 \text { min, C: Selective- clamp, D: Off-clamp, ASA: American society of anesthesiologists, BMI: Body mass index, CAD: Coronary artery disease, DM: } \\
\text { Diabetes Mellitus, HT: Hypertension, RNS: RENAL Nephrometry score, WIT: Warm ischaemia time, *: Continuous data are expressed as mean } \pm \text { standard deviation and } \\
\text { categorical data as number (\%), a,b,c: Different lowercase letters show a significant difference between groups by One-Way ANOVA followed by Bonferroni Post-hoc test } \\
(p<0.05), \mathrm{mm} \text { : Millimeter, kg: Kilogram }\end{array}$} \\
\hline
\end{tabular}

\begin{tabular}{|c|c|c|c|c|c|}
\hline Variable ${ }^{* *}$ & $A(n=33)$ & $B(n=202)$ & $C(n=16)$ & $D(n=36)$ & $\mathrm{p}$ \\
\hline Operation time, min & $123.76 \pm 35.1^{\mathrm{a}}$ & $92.72 \pm 30.5^{\mathrm{b}}$ & $100.3 \pm 18.5^{\mathrm{ab}}$ & $79.72 \pm 30.6^{b}$ & 0.001 \\
\hline WIT, min & $23.82 \pm 2.6$ & $12.94 \pm 3.8$ & $13.31 \pm 4.1$ & 0 & - \\
\hline Blood loss, mL & $136.36 \pm 76.3$ & $140 \pm 83.8$ & $118.13 \pm 54.8$ & $165.9 \pm 87.3$ & 0.570 \\
\hline PSM & 0 & $4(2)$ & 0 & 0 & - \\
\hline Intraoperative complications rate & $2(6.1)$ & $4(2)$ & $2(12.5)$ & 0 & 0.085 \\
\hline Postoperative complication rate & $6(18.2 \%)$ & $18(8.9 \%)$ & $1(6.3 \%)$ & $3(8.3 \%)$ & 0.443 \\
\hline Relative eGFR change $(\%)^{*}$ & $9.3^{\mathrm{a}}$ & $7.5^{\mathrm{a}}$ & $5^{\mathrm{b}}$ & $3.7^{\mathrm{b}}$ & 0.001 \\
\hline \multicolumn{6}{|c|}{$\begin{array}{l}\text { A: WIT }>20 \text { min, B: WIT } \leq 20 \text { min, C: Selective-clamp, D: Off-clamp, a,b,c: Different lowercase letters show a significant difference between groups by One-Way ANOV } \\
\text { followed by Bonferroni Post-hoc test ( } p<0.05) \text {, PSM: Positive surgical margin, }{ }^{*} 1 \text { year after LPN, }{ }^{*} \text { : Continuous data are expressed as mean } \pm \text { standard deviation an } \\
\text { categorical data as number }(\%) \text {, min: Minute, WIT: Warm ischaemia time, eGFR: Estimated glomerular filtration rate }\end{array}$} \\
\hline
\end{tabular}


The main effect of groups on eGFR was statistically significant $(p<0.001)$. The main effect of time on eGFR was also statistically significant, and in the preoperative period, eGFR was higher than others, except in the first year $(p<0.001)$. Nevertheless, group and time interaction was not statistically significant $(p=0.960)$. The group, time, and group-time effect on eGFR are summarised in Table 3.

When the change in eGFR ( $\triangle$ eGFR) between the groups over time was evaluated, the main effects of the groups were statistically significant $(p<0.001)$. Although there was no statistical difference between groups $D$ and $C$, these two groups were significantly different from the other groups. There was no statistical difference between group A and group B. The time effect was also statistically significant $(p<0.001)$. There was no difference between the postoperative first-day $\triangle \mathrm{eGFR}$ and the first-month $\triangle$ eGFR. Similarly, $\triangle$ eGFR values in the sixth month was non-significantly different $(p>0.05)$ from $\triangle$ eGFR in the first year. However, other time comparisons were statistically significant. Group-time interaction was not statistically significant $(p=0.960)$. The group, time and group-time effect on $\triangle \mathrm{eGFR}$ are summarised in Table 4.

\section{Discussion}

The present study revealed that zero-ischaemia groups ( $C$ and D: 8.71 vs. $5.49 \mathrm{~mL} /$ minute $/ 1.73 \mathrm{~m}^{2}$ ) have an advantage in terms of eGFR decline ( $\triangle$ eGFR) compared to the global ischaemia groups ( $A$ and $B: 10.38$ vs $8.98 \mathrm{~mL} /$ minute $/ 1.73 \mathrm{~m}^{2}, \mathrm{p}<0.001$ ) at one year after LPN. The percentage of relative eGFR decline 1 year after LPN was statistically different in the zero-ischaemia groups (C and D: $5 \%$ and 3.7\%) compared to the global ischaemia groups ( $A$ and $B: 9.3 \%$ and $7.5 \%, p=0.001$ ).
Baseline RF and volume of preserved renal parenchyma are the significant determiners of functional recovery following PN. Besides, prolonged WIT negatively also affects postoperative RFal recovery (12). It is difficult to detect the effect of PN on total RF in patients with contralateral healthy kidney because of the compensatory role played by the unaffected kidney. Renal scintigraphy, also known as renal scans, in combination with eGFR gives the most accurate results in assessing the RF of affected kidney after PN (13). However, preoperative and postoperative evaluation of patients who underwent PN by scintigraphy is a difficult, and is a non-cost effective approach in clinical practice. Therefore, total eGFR has been widely employed as a surrogate measure of RF. Furthermore, the necessity of evaluation by scintigraphy may be questioned in patients with uncomplicated renal mass, normal contralateral kidney, or whose WIT is below recommended time levels. Additionally, it would be more accurate if the amount of preserved renal parenchyma following PN was calculated alongside the tumour size and WIT. However, there is no adequate technical infrastructure to precisely estimate the preserved renal parenchyma. This situation is therefore considered a limitation of the present study.

We routinely evaluated the RF of patients who underwent LPN using the CKD-EPI equation before and after the surgery. CKDEPI equation, compared to the Modification of Diet in Renal disease equation, has been associated with more accurate outcomes in patients with eGFR levels $>60 \mathrm{~mL} /$ minute (5). In this study, total eGFR values were used for the interpretation of functional evaluation. Furthermore, patients who underwent $\mathrm{PN}$, and with normal contralateral kidney, are expected to have a $10 \%$ reduction in $\mathrm{RF}$ or maintain $90 \%$ of total $\mathrm{RF}$ in general. These criteria was recently used in the evaluation of surgical success in functional aspects (14).

\begin{tabular}{|c|c|c|c|c|c|}
\hline eGFR & $A(n=33)$ & $B(n=202)$ & $C(n=16)$ & $D(n=36)$ & Total \\
\hline Baseline & $101.31 \pm 28.45$ & $104.98 \pm 28.17$ & $108.75 \pm 23.01$ & $98.81 \pm 26.97$ & $104.00 \pm 27.77^{a}$ \\
\hline $1^{\text {st }}$ month & $84.08 \pm 18.11$ & $90.42 \pm 19.74$ & $99.42 \pm 15.25$ & $93.61 \pm 23.21$ & $90.59 \pm 19.98^{b}$ \\
\hline $6^{\text {th }}$ months & $88.1 \pm 20$ & $94.05 \pm 21.53$ & $100.8 \pm 15.42$ & $94.65 \pm 23.73$ & $93.81 \pm 21.41^{\mathrm{b}}$ \\
\hline
\end{tabular}

\begin{tabular}{|c|c|c|c|c|c|}
\hline$\triangle \mathrm{eGFR}$ & $A(n=33)$ & $B(n=202)$ & $C(n=16)$ & $D(n=36)$ & Total \\
\hline 1 & $19.82 \pm 16.29$ & $17.72 \pm 14.76$ & $11.13 \pm 12.18$ & $8.42 \pm 11.42$ & $16.43 \pm 14.79^{c}$ \\
\hline 3 & $13.21 \pm 12.24$ & $10.94 \pm 9.73$ & $7.94 \pm 11.1$ & $4.16 \pm 5.8$ & $10.18 \pm 10.01^{\mathrm{ab}}$ \\
\hline 4 & $10.38 \pm 10.49$ & $8.98 \pm 9.32$ & $6.44 \pm 8.36$ & $4.19 \pm 5.62$ & $8.4 \pm 9.17^{a}$ \\
\hline
\end{tabular}


Standard PN requires transient ischaemia to obtain a higher quality of vision, with a relatively bloodless field during tumour resection and renorrhaphy. However, it is well established that postoperative renal functional preservation is closely associated with preserved nephron volume, as well as ischaemic damage on the remaining renal parenchyma (15). A variety of experimental and clinical investigations has focused on this issue. Nevertheless, the optimal threshold for ischaemia time to preserve postoperative RF following PN is still controversial (12). Moreover, recent studies suggest that the optimal threshold of WIT does not exceed 20 minute $(16,17)$. On the other hand, selective-clamp and off-clamp techniques have been demonstrated to minimise or eliminate ischaemia-related kidney injury. However, the indication of these techniques that refer to zero-ischaemia in patients with contralateral healthy kidney is debatable as a result of the increased risk of perioperative blood loss, and technical challenges, thus requiring a considerable PN experience (18).

Although there is an increase in the amount of EBL in studies comparing the off-clamp technique with the global ischaemic technique, it is stated that the complications are similar, while the functional results are better. However, selection bias, heterogeneity of patients and differences in surgical techniques are the major concerns that may affect the final outcomes.

Smith et al. (19) compared 116 clamped and 192 off-clamp PNs in terms of perioperative, functional and oncological outcomes. While all patients were managed by open PN in the off-clamp group, more than half of the patients underwent laparoscopic or robotic PN in the clamped group. It has found that the median tumour size (3 vs $2.8 \mathrm{~cm}, \mathrm{p}=0.002)$, EBL (500 vs $200 \mathrm{~mL}$, $\mathrm{p}<0.001)$, perioperative transfusion rate ( $42 \%$ vs $23 \%, \mathrm{p}=0.001)$ and OT ( 226.5 vs $192 \mathrm{~min}, \mathrm{p}=0.001)$ were significantly higher in the off-clamp group when compared to the clamped group. The authors also reported that eGFR decline was significantly higher in the clamped group compared to the off-clamp group $(12.3 \%$ vs $9.8 \%, p=0.037$ ) after 1 year of follow-up.

Kopp et al. (20) compared 164 clamped with 64 off-clamp open PNs in patients with bilateral kidney. The off-clamp group had higher EBL ( 300 vs $200 \mathrm{~mL}, \mathrm{p}=0.001$ ) and lower rate of de novo CKD (12.5 vs $24.4 \%, p=0.049$ ) following PN when compared to the clamped group. However, the complication rate was nonsignificantly $(p>0.05)$ different between the groups $(14.2 \%$ vs $14.1 \%, p=0.43)$. In their study, the significant drawbacks was that tumour complexity (RENAL score 6.9 vs $6.4, p=0.02$ ) was higher in the clamped group, and standard surgical technique was not employed.

Simone et al. (21) compared the functional outcomes of 472 off-clamps versus 157 on-clamp open PNs after Propensity score matching analysis. The authors found that the on-clamp group had a higher probability of developing $\geq 3 b$ CKD when compared to the off-clamp group. The risk of developing CKD was 7.3 fold higher in the on-clamp group during follow-up.

Browne et al. (22) evaluated preioperative and postoperative outcomes of 43 patients who underwent LPN. They used a YAG laser or vessel sealing device for tumour resection and tumour bed ablation. The authors found that the mean tumour size was $28.2 \mathrm{~mm}$, OT was $172 \mathrm{~min}$ and EBL was $341 \mathrm{~mL}$. The relative change in eGFR was reduce by $2.8 \% 6$ months after the surgery. In a recent systematic review of 23 retrospective and 2 prospective cohort studies, early postoperative eGFR decline, long-term eGFR change and percentage of change were significantly lower in off-clamp patients when compared to the on-clamp cohorts (23). In our study, the amount of preoperative bleeding in the off-clamp group was non-significantly different from that of the other groups. Surgical time was also significantly shorter than in other groups compared to the off-clamp group. In terms of functional results, the decrease in GFR was minimum in the off-clamp group in both early postoperative period and during follow-up.

Selective arterial clamping is another zero-ischaemia PN method introduced for the first time by Gill et al. (24). In this technique, ischaemia is created in the region to be excised, while the remaining part of the kidney is protected from ischaemia. NIRF/ ICG-guided minimally invasive PN is one of the important advancement in imaging systems. Although not widely used, it is considered as a useful technique for specifying tumour margins and preserving normal parenchyma (25). Selective-clamping is said to have similar functional outcomes with the off-clamp $\mathrm{PN}$, however, it has more advantage in terms of preservation of RF when compared to the global ischaemia technique (2). The results of the present study is consistent with that of previous studies in literature where postoperative functional outcomes were similar between selective-clamp and off-clamp group, and selective-clamp better than the global ischaemic groups.

\section{Study Limitations}

The limitations of our study was due to retrospective design, single-centredness, single-surgeon experience and different distribution of patients within the groups. However, singlesurgeon experience may be an advantage for the standardisation of laparoscopy technique. Since patients with contralateral healthy kidney were included in the study, we presumed that the negative effect of selection bias on final functional outcomes would decrease.

\section{Conclusion}

Reduction or elimination of ischaemia time in LPN has positive effects on the preservation of RF in the postoperative period. If global ischaemia technique is preferred, tumour resection and renorraphy should be performed in less than 20 minutes. Prospective studies are however required to validate this study.

\section{Acknowledgements}

Publication: The initial outcomes of this study have been accepted and presented in the "14 th Uro Oncology Congress", which was held on November 6-10, 2019, Antalya, as an oral presentation (SS053).

Contribution: There is not any contributors who may not be listed as authors.

Conflict of Interest: No conflict of interest was declared by the authors.

Financial Disclosure: The authors declared that this study received no financial support. 


\section{Ethics}

Ethics Committee Approval: The study has been approved by the Ondokuz Mayıs University Clinical Research Ethics Committee (OMU KAEK 14.02.2019/151).

Informed Consent: Written informed consent were obtained from all patients included in the study.

Peer-review: Externally peer-reviewed

\section{Authorship Contributions}

Critical Review: E.Ö., Y.K.Y., Ş.S., Concept: M.N.M., E.Ö., Design: M.N.M., E.Ö., Data Collection or Processing: M.N.M., E.Ö., M.G., Y.K.Y., Ş.S., Analysis or Interpretation: M.N.M., E.Ö., M.G., Y.B., Y.K.Y., S..S., Literature Search: M.N.M., M.G., Y.B., Writing: M.N.M., E.Ö., M.G., Y.B., Y.K.Y., Ş.S.

\section{References}

1. Ljungberg B, Albiges $L$, Abu-Ghanem $Y$, et al. European association of urology guidelines on renal cell carcinoma: the 2019 update. Eur Urol 2019;75:799-810.

2. Marconi L, Desai MM, Ficarra V, et al. Renal Preservation and Partial Nephrectomy: Patient and Surgical Factors. European urology focus 2016;2:589-600.

3. Thompson RH, Lane BR, Lohse CM, et al. Every minute counts when the renal hilum is clamped during partial nephrectomy. Eur Urol 2010;58:340-345.

4. Ng AM, Shah PH, Kavoussi LR. Laparoscopic partial nephrectomy: A narrative review and comparison with open and robotic partial nephrectomy. J Endourol 2017;31:976-984.

5. Levey AS, Stevens LA. Estimating GFR using the CKD Epidemiology Collaboration (CKD-EPI) creatinine equation: more accurate GFR estimates, lower CKD prevalence estimates, and better risk predictions. Am J Kidney Dis 2010;55:622-627.

6. Kutikov A, Uzzo RG. The R.E.N.A.L. nephrometry score: a comprehensive standardized system for quantitating renal tumor size, location and depth. J Urol 2009;182:844-853.

7. Clavien PA, Barkun J, de Oliveira ML, et al. The Clavien-Dindo classification of surgical complications: five-year experience. Ann Surg 2009;250:187-196.

8. Singh D, Rubenstein M, Gill IS. Laparoscopic partial nephrectomy. J Endourol 2005; 19:451-455.

9. Gill IS, Rassweiler JJ. Retroperitoneoscopic renal surgery: our approach. Urology 1999;54:734-738.

10. Yamasaki T, Tamada S, Kato $M$, et al. Near infrared fluorescence imaging system for laparoscopic partial nephrectomy. Can J Urol 2018;25:9606-9613.

11. Gill IS, Patil MB, Abreu AL, et al. Zero ischemia anatomical partial nephrectomy: a novel approach. Arab J Urol 2012;187:807-814.
12. Volpe A, Blute ML, Ficarra V, et al. Renal Ischemia and Function After Partial Nephrectomy: A Collaborative Review of the Literature. Eur Urol 2015;68:61-74.

13. Porpiglia $\mathrm{F}$, Autorino $\mathrm{R}$, Fiori $\mathrm{C}$, et al. The impact of $\mathrm{T} 1$ renal tumor characteristics on baseline renal function in patients undergoing partial nephrectomy: A renal scan based objective assessment. Eur J Surg Oncol 2017;43:1598-1602.

14. Mir MC, Campbell RA, Sharma N, et al. Parenchymal volume preservation and ischemia during partial nephrectomy: functional and volumetric analysis. Urology 2013;82:263-268.

15. Zabell JR, Wu J, Suk-Ouichai C, Campbell SC. Renal Ischemia and Functional Outcomes Following Partial Nephrectomy. Urol Clin North Am 2017;44:243-255.

16. Porpiglia F, Bertolo R, Amparore D, Cristian Fiori. Margins, ischaemia and complications rate after laparoscopic partial nephrectomy: impact of learning curve and tumour anatomical characteristics. BJU Int 2013;112:1125-1132.

17. Bianchi L, Schiavina R, Borghesi $M$, et al. Which patients with clinical localized renal mass would achieve the trifecta after partial nephrectomy? The impact of surgical technique. Minerva Urol Nefrol 2020;72:339-349.

18. Simone G, Gill IS, Mottrie A, et al. Indications, techniques, outcomes, and limitations for minimally ischemic and off-clamp partial nephrectomy: a systematic review of the literature. Eur Urol 2015;68:632-640.

19. Smith GL, Kenney PA, Lee Y, Libertino JA. Non-clamped partial nephrectomy: techniques and surgical outcomes. BJU Int 2011; 107:1054-1058.

20. Kopp RP, Mehrazin R, Palazzi K, et al. Factors affecting renal function after open partial nephrectomy-a comparison of clampless and clamped warm ischemic technique. Urology 2012;80:865-870.

21. Simone G, Capitanio U, Tuderti G, et al. On-clamp versus off-clamp partial nephrectomy: Propensity score-matched comparison of longterm functional outcomes. Int J Urol 2019;26:985-991.

22. Browne $C$, Lonergan $P$, Bolton $E$, et al. A single centre experience of zero-ischaemia laparoscopic partial nephrectomy in Ireland. Ir J Med Sci 2017;186:1023-1026.

23. Deng W, Liu X, Hu J, et al. Off-clamp partial nephrectomy has a positive impact on short- and long-term renal function: a systematic review and meta-analysis. BMC Nephrol 2018;19:188.

24. Gill IS, Patil MB, de Castro Abreu AL, et al. Zero ischemia anatomical partial nephrectomy: a novel approach. The Journal of urology 2012;187:807-814.

25. Cacciamani GE, Shakir A, Tafuri A, et al. Best practices in nearinfrared fluorescence imaging with indocyanine green (NIRF/ICG)guided robotic urologic surgery: a systematic review-based expert consensus. World J Urol 2020;38:883-896. 